\title{
The effect of edge orientation and movement direction on the apparent misalignment of collinear bars
}

\author{
R. H. DAY, E. J. STECHER, and ANDREA L. PARKER \\ Monash University, Clayton, Victoria, Australia
}

\begin{abstract}
Four experiments on apparent misalignment of oblique collinear bars are reported. The data from the first three experiments showed that the misalignment was slight but significant when no direction other than that of the bars themselves was delineated and about double when the vertical was delineated by movements of the adjustable bar. When the vertical was delineated by both these movements and the ends of the bars (or by vertical parallel lines), the misalignment was more than six times greater. Conversely, it was reduced when the bar ends delineated the vertical and bar movements delineated the direction at right angles to the bars. The data from a fourth experiment showed that the inner pair of edges were closely involved in apparent misalignment and that the outer ends were not. The relationship between the misalignment effect with bars and the Poggendorff figure (oblique lines separated by parallels), the delineation of direction by edges and movement, and the implications of these data for an explanation are discussed.
\end{abstract}

In two recent experiments (Day \& Stecher, 1992), it was shown that collinear oblique bars, like those in Figure 1A, appear to be misaligned and that the effect is much greater when the inner ends have a vertical chamfer as in Figure 1B. Apparent misalignment in Figure 1B proved to be about the same as that in Figure $1 \mathrm{C}$, a version of the conventional Poggendorff figure. These results were explained in terms of a compromise in perception between the alignment of bars in their oblique axis and their displacement from each other, that is, their nonalignment in the vertical axis. This explanation had been proposed earlier (Day, 1989) for the illusion in the conventional parallel-line figure. The essence of this explanation is that whereas the oblique transversals are aligned in their own axis, they are displaced from each other relative to the vertical parallels that they intersect.

It is proposed that this large displacement (i.e., misalignment) in one delineated direction, combined with the alignment in the other direction, results in a slight apparent misalignment. In other words, there is a perceptual compromise between the two states of alignment. In the case of the bars, it was noted that one bar was always moved vertically during adjustments to apparent alignment. The misalignment effect was therefore held to be due to the physical displacement of the bars from each other relative to the vertical delineated by movements of the adjustable bar in Figure 1A, by movements and the

The assistance of Mike Durham, Vladimir Kohout, and Rosemary Williams in computer programming, photography, and illustration, respectively, is gratefully acknowledged. Correspondence should be addressed to R. H. Day, Department of Psychology, Monash University, Clayton, Victoria, 3168, Australia. chamfered vertical ends of the bars in Figures 1B, and by movements and the vertical edges of the rectangle in Figure 1C.

The four experiments we report here were primarily concerned with the role and relative effectiveness of movement and edges in determining apparent misalignment and, secondarily, with the perceptual-compromise explanation of apparent misalignment, summarized above. The first experiment was designed to establish the size of the misalignment effect when the vertical was delineated by movement alone, as well as by movement in conjunction with either the ends of the bars or the extended parallel lines (see Figure 2). In the second experiment, which was essentially a control for the first, the vertical was not delineated by movement, bar ends, vertical lines, or any other means. The third experiment was concerned with the magnitude of the misalignment effect when bar movement and bar ends delineated the vertical or the axis at right angles to the bars in either correspondence or noncorrespondence with each other (see Figure 3). The purpose of the fourth experiment was to ascertain whether the inner and outer vertical ends of the bars contribute equally or unequally to the misalignment effect.

\section{EXPERIMENT 1}

The three stimulus figures used to establish the effects of delineation of the vertical by bar movement, bar ends, and vertical parallels are shown in Figure 2. In all three figures, the bar on the right moved vertically. The outer ends of all bars were semicircular and therefore did not delineate any specific direction. In Figure 2A, the inner ends were also semicircular. In Figure $2 B$, they were chamfered vertically (i.e., at $45^{\circ}$ to the axis of the bars) 


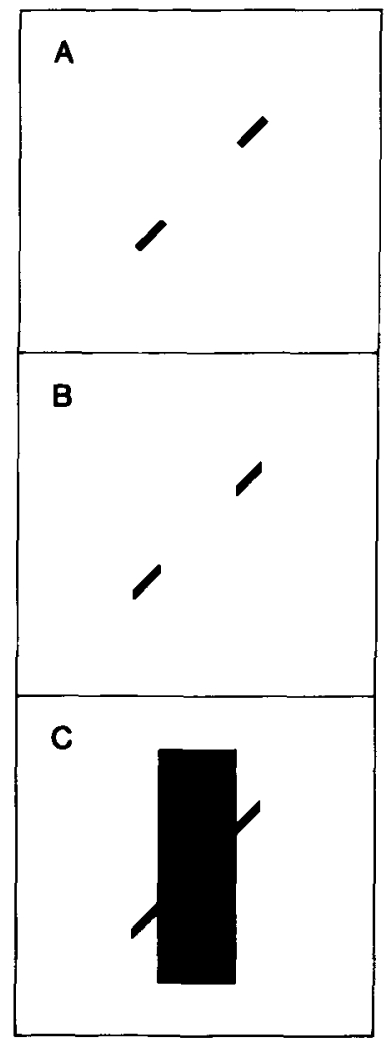

Figure 1. Apparent misalignment of collinear oblique bars with the bar ends at right angles to the bars (A), vertical (B), and abutting onto vertical lines (C).

and parallel. In Figure $2 \mathrm{C}$, they intersected the vertical lines.

\section{Method}

Subjects. There were 12 undergraduate subjects $(9$ women and 3 men), all of whom volunteered to participate and were paid for doing so.

Apparatus. The apparatus was the same as that described earlier (Day \& Stecher, 1992). The figures were presented on the $27.5 \times$ $20.4 \mathrm{~cm}$ screen of a personal computer adapted for the purpose. The viewing distance was $114 \mathrm{~cm}$ (i.e, $1 \mathrm{~cm}$ at the screen subtended $0.50^{\circ}$ at the eye). The computer generated the figures, presented them in random order, varied the starting position of the adjustable bar on the right, and recorded, averaged, and stored individual scores. The adjustable bar could be moved vertically by pressing one of two directionally marked keys. A third key brought up the next figure in the sequence. To eliminate any possibility that the misalignment effect would be influenced by the contours of the screen, a mask with a circular aperture $(17 \mathrm{~cm}$ in diameter) was placed over the screen. Thus, the stimulus figures were presented in a circular field.

Stimulus figures. The bars were $25 \times 5 \mathrm{~mm}$ and separated by $25 \mathrm{~mm}$ along their central axis when aligned. In all figures, it was the bar on the right that could be moved for adjustments to apparent alignment. The vertical parallel lines were approximately $0.4 \mathrm{~mm}$ wide and extended from the top to the bottom of the circular field. The oblique distance between the bars was also $25 \mathrm{~mm}$.

Procedure. The subjects moved the adjustable bar until it appeared to be perfectly aligned with the other. Each figure was presented four times, with the initial position of the adjustable bar al- ternating between $18.7 \mathrm{~mm}$ above the position of true alignment and $18.7 \mathrm{~mm}$ below it. All four adjustments to apparent alignment for one figure were completed before the next figure was presented. The score was the mean vertical difference in millimeters between apparent and true alignment. Scores in the direction to compensate for the Poggendorff effect were treated as positive; those in the opposite direction were treated as negative.

\section{Results and Discussion}

The mean misalignment scores and their standard deviations are shown in Figure 2. It can be seen that the means for Figures $2 \mathrm{~B}$ and $2 \mathrm{C}$ were about the same and about three times the size of that for Figure 2A. Separate $t$ tests showed that the means were significantly different from zero (Figure 2A, $p<.02$; Figures $2 \mathrm{~B}$ and $2 \mathrm{C}, p<$ .001 ). A one-way analysis of variance (ANOVA) showed that the stimulus configuration was a significant determinant of misalignment effects $[F(1,11)=16.07, p<$ $.001]$. A Newman-Keuls analysis showed that the means for Figures $2 \mathrm{~B}$ and $2 \mathrm{C}$ were not significantly different from each other $(p>.05)$ but that both were significantly greater than that for Figure 2A $(p<.01)$. Two conclusions may be drawn from these outcomes. The first is that delineation of the vertical by movements of one bar during its adjustment to apparent alignment with the other gives rise to a marked misalignment effect. Second, the

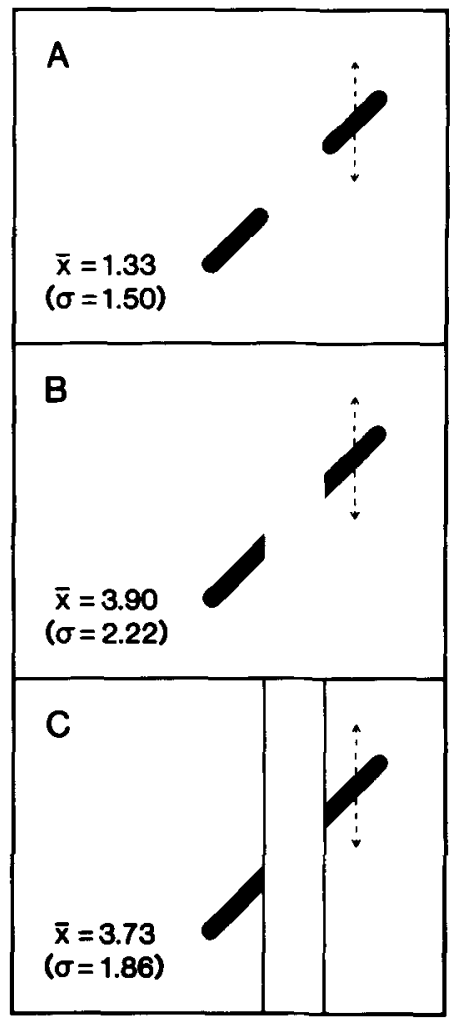

Figure 2. The three stimulus figures for Experiment 1, together with their mean misalignment effects (in millimeters) and standard deviations. The vertical movements of the bar on the right during adjustments to apparent alignment are also indicated. 
effect is almost tripled in size when delineation of the vertical by these movements is combined with delineation by either bar ends or parallel lines. However, before concluding that vertical delineation by movement alone generates a misalignment effect in Figure $1 \mathrm{~A}$, it was considered prudent to ascertain whether the effect occurs in the absence of any such delineation.

\section{EXPERIMENT 2}

In the second experiment, which was a control for the first, there was no delineation of direction other than that of the $45^{\circ}$ oblique bars themselves. ${ }^{1}$ Delineation of the vertical by the ends of the bars was eliminated, as in Experiment 1 , by making the ends semicircular, and delineation by movements of the adjustable bar was eliminated by means of the stepwise staircase procedure. The field in which the figures were presented was again circular, as in Experiment 1.

\begin{abstract}
Method
Subjects. Another group of 12 undergraduate subjects $(6$ men and 6 women) served as subjects.

Apparatus and stimulus figure. The apparatus was the same as that in Experiment 1, and the stimulus figure was the same as that in Figure 2A.

Procedure. The staircase procedure described by Cornsweet (1962) was used to present the stimulus figure. This method involved a step-by-step presentation of differences in an object property-in this case, the state of alignment of two stimulus elements. Judgments were restricted to small differences near the threshold for apparent misalignment. There were two independent randomly interleaved staircase series. In one series, the bar on the right moved obliquely downward in $0.37-\mathrm{mm}$ steps from a position obviously too high for alignment; in the other series, the same bar moved upward from a position obviously too low. The subjects reported only whether the bar on the right was too high or too low for alignment with that on the left. The subjects were required to close their eyes between judgments so that the small stepwise movements could not be seen. The procedure continued until 12 changes of response from "too high" to "too low," or vice versa, were recorded. The score was always the vertical distance between the mean position of the last 10 points of change and the point of true alignment. A positive mean again indicated compensation for a misalignment effect in the direction of the Poggendorff illusion.
\end{abstract}

\section{Results and Discussion}

Eleven of the 12 scores were positive. The mean score was $0.6 \mathrm{~mm}(S D=0.53)$. A $t$ test showed that this mean was significant $[t(11)=3.92, p<.01]$. Thus, although the vertical was not delineated, a small but reliable misalignment effect occurred. This effect was notably less than that of $1.33 \mathrm{~mm}$ in Experiment 1 in which the vertical was delineated by movements of the adjustable bar. A possible basis for this small but significant effect without delineation of the vertical is considered in the General Discussion section below.

Since the outcome of this experiment strongly implicates delineation of the vertical by movement of the adjustable bar as a significant factor in the apparent misalignment of the two bars in Experiment 1, it is relevant to consider the data on the Poggendorff figure reported by Mori (1981). In his experiments, Mori investigated the effects of vertical movement on apparent misalignment in the conventional, parallel-line Poggendorff figure. Both oblique transversals moved in unison (not as here where one oblique element was moved during adjustments). It was found that for high-velocity movement $(21.2 \% \mathrm{sec})$ the misalignment was reduced by about $60 \%$, and for low velocity movement $(1.3 \% \mathrm{sec})$ the misaslignment was reduced by about $34 \%$. However, the conditions for this earlier experiment and those for the present Experiment 1 were very different. Mori's oblique elements were lines rather than broad bars. Furthermore, movement of the bar was initiated and controlled by the subject rather than occurring independently. Subjects could move the bar in single steps and stop movement altogether if they wished to judge whether or not the bars seemed aligned. As has been noted, the misalignment effect in Figure 2A without movement of the adjustable bar was substantially less than that in the previous experiment with movement. It is clear, therefore, that subjectcontrolled movements of one bar during adjustments to apparent alignment and externally controlled movement of both bars in unison have very different effects on apparent alignment.

\section{EXPERIMENT 3}

In Experiment 1, the size of the misalignment effect when movements of the adjustable bar alone delineated the vertical was more than doubled when these movements together with the ends of the bars did so. As pointed out in the introduction, the oblique bars are vertically displaced from each other but are not displaced relative to their own axes. In other words, the bars are misaligned relative to the vertical but aligned relative to the axes of the bars. The bars are also aligned relative to oblique axes at right angles to the bars. It could therefore be expected that when both movements of the adjustable bar and the edges at the ends of the bars delineated this oblique axis, no misalignment effect would occur. This situation is the same in principle as the conventional Poggendorff figure with collinear, oblique transversals at right angles to oblique parallels. In this arrangement, apparent misalignment does not occur (Weintraub, Krantz, \& Olson, 1980). The question arises as to the size of the misalignment effect when the movement of the adjustable bar delineates one of these two directions, and the ends of the bars delineates the other. The answer to this question is of interest for two reasons. First, it stands to reveal whether the movements and bar ends interact in determining apparent misalignment and, second, whether one of these two modes of delineation (movement vs. bar ends) is more effective, that is, more "salient" than the other, as a determinant of apparent misalignment.

The four conditions of delineation in this experiment are shown in Figure 3. In one condition (A), both the direction of movement and the ends of the bars delineated the vertical; in another (D), both delineated the $45^{\circ}$ 
oblique direction at right angles to the bars. In the other two conditions ( $B$ and $C$ ), movements delineated the oblique direction and bar ends delineated the vertical and vice versa.

\section{Method}

Subjects. Another group of volunteer undergraduates ( 8 women and 4 men) participated.

Apparatus and Procedure. The apparatus and procedure were the same as those for Experiment 1 except that in this experiment the mask with the circular aperture was not used. ${ }^{2}$

Stimulus figures. The stimulus figures with their four combinations of movement direction and bar-end orientation are shown in Figure 3. The bars were again $25 \times 5 \mathrm{~mm}$, and the separation between them was $25 \mathrm{~mm}$ when they were aligned.

\section{Results and Discussion}

The mean misalignment effects and their standard deviations are shown in Figure 3. The effect in Figure 3A, in which both movements and bar ends were vertical, was relatively large, that in Figure $3 \mathrm{D}$ in which both were oblique almost zero. The effect in Figure 3B, in which movements were oblique and bar ends were vertical, was intermediate. The effect in Figure 3C, in which movements were vertical and bar ends were oblique, was also intermediate but much less than that in Figure 3B. Separate $t$ tests showed that the means for Figures $3 A$ and $3 B$ were significantly different from zero [Figure $3 \mathrm{~A}, t(11)$ $=5.54, p<.001$; Figure 3B, $t(11)=4.88, p<.001]$

A

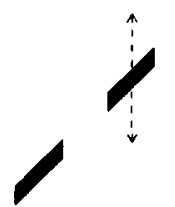

$\overline{\mathrm{x}}=3.47$

$(\sigma=2.17)$
B

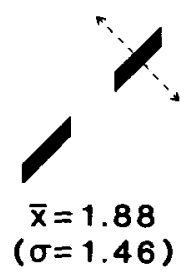

C

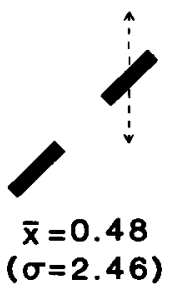

but that those for Figures 3C and 3D were not [Figure 3C, $t(11)=0.68, p>.05 ;$ Figure $3 \mathrm{D}, t(11)=0.26, p>$ .05]. A two-way ANOVA in which the factors were the orientation of the ends of the bars $(\mathrm{O})$ and the direction of movement (D) of the adjustable bar showed that both factors were significant $[0, F(1,11)=65.91, p<.001$; $\mathrm{D}, F(1,11)=10.74, p<.01]$. The interaction between these two factors was also significant $[F(1,11)=9.59$, $p<.05]$.

The most interesting outcome from Experiment 3 is the difference between the misalignment effects when the directions delineated by the bar ends and movements of the adjustable bar were in conflict. When bar ends were vertical and movements of the adjustable bar were at right angles to the bars (Figure 3B), the effect was half that in Figure 3A but still significant. When this situation was reversed so that the bar ends were oblique and at right angles to the bars and bar movements were vertical, the effect was negligible. This asymmetry between the effects of delineation by movement and edges suggests that, of the two modes of delineation of direction, edges are more effective than movement. This issue is considered further below.

\section{EXPERIMENT 4}

From the standpoint of the perceptual-compromise explanation of apparent misalignment, it would seem of little or no consequence whether the direction relative to which the bars are misaligned is delineated by the inner ends of the bars, the outer ends, or both. The only essential requirement for this explanation is that this direction should be delineated by some means, including the edges at the ends of bars, extended edges or lines, or movement. In these terms, it can be expected that misalignment effects will occur when the vertical is delineated by either the inner or the outer ends of the bars, or by both. This expectation was tested in Experiment 4, using the four stimulus figures shown in Figure 4. The ends of the bars were both semicircular (A), both vertical (D), the inner ends vertical and the outer ends semicircular (B), and the inner ends semicircular and the outer ends vertical (C). The direction of movement of the adjustable bar was oblique (at right angles to the bars) in all four figures, as also indicated in Figure 4. It was expected that the misalignment effect would be negligible in Figure 4A and significant in Figures 4B, 4C, and 4D. No predictions were made about the relative magnitude of the effects in the latter three figures. However, there was no reason in terms of the perceptual-compromise explanation (as currently formulated) to expect that they would be different.

\section{Method}

Subjects. There were again 12 volunteer subjects $(7$ men and 5 women), all of whom were undergraduates.

Apparatus and Procedure. The apparatus and procedure were the same as those for Experiment 3.

Stimulus figures. The dimensions of the bars $(25 \times 5 \mathrm{~mm})$ and their separation along their central axis when aligned $(25 \mathrm{~mm})$ were the same as those in Experiment 3.

\footnotetext{
Figure 3. The four stimulus figures for Experiment 3, together with their mean misalignment effects (in millimeters) and standard deviations. The directions of movements of the adjustable bars are also indicated.
} 
A

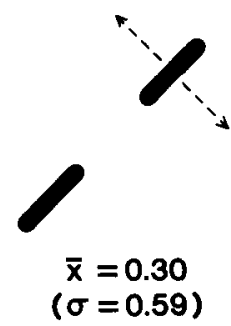

C

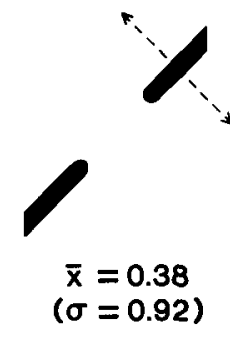

B

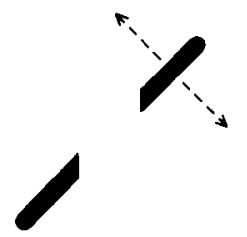

$\bar{x}=1.60$

$(\sigma=0.87)$

D

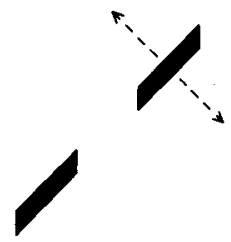

$\overline{\mathrm{x}}=1.93$

$(\sigma=0.86)$

Figure 4. The four stimulus figures for Experiment 4, together with their mean misalignment effects (in millimeters) and standard deviations. The oblique direction of movements of the adjustable bar is also indicated.

\section{Results and Discussion}

The mean misalignment scores and their standard deviations are shown in Figure 4 . It can be noted that the means for the two figures with semicircular inner ends (Figures 4A and 4C) were relatively slight and about the same, and those for the two figures with vertical inner ends (Figures 4B and 4D) were relatively large and also about the same. It can also be noted that the mean for Figure $4 \mathrm{D}$ was about the same size as that for the same configuration (Figure 3B) in Experiment 3. Independent $t$ tests showed that whereas the mean scores for Figures $4 B$ and $4 D$ were significantly different from zero [Figure $4 \mathrm{~B}, t(11)=6.34, p<.001$; Figure $4 \mathrm{D}, t(11)=$ $7.78, p<.001$ ], those for Figures $4 \mathrm{~A}$ and $4 \mathrm{C}$ were not [Figure 4A, $t(11)=1.75, p>.05$; Figure $4 \mathrm{C}, t(11)=$ $1.44, p>.05]$. A two-way ANOVA in which the main factors were the form of the inner bar ends (vertical or semicircular), and the accord between the inner and outer ends of the bars (same or different) showed that the first of these factors was significant but the second was not [form, $F(1,11)=44.40, p<.001$; accord, $F(1,11)=$ $0.49, p>.05]$. The interaction between these factors also failed to reach significance $[F(1,11)=2.17, p>.05]$. - It is quite clear from these outcomes that, for the stimulus figures in Figure 4, only the inside vertical edges of the bars are involved in the misalignment effect, and that the outside edges do not contribute at all. When the

inside edges were vertical the misalignment effect was relatively large, regardless of whether the outside edges were vertical or semicircular. Likewise, there was no misalignment effect when the inside edges of the bars were semicircular, regardless of whether the outside edges were vertical or semicircular. These outcomes cannot be readily explained in terms of the perceptual-compromise explanation as it stands.

\section{GENERAL DISCUSSION}

The data from the four experiments can be summarized as follows. A small but reliable misalignment effect occurred with oblique bars when the only delineated direction was that of the bars themselves (Experiment 2), a much greater effect when the vertical was delineated by movements of the adjustable bar, and a still greater effect when the vertical was delineated by this movement together with either the ends of the bars or the parallel lines with which the adjustable bars intersect (Experiment 1). The misalignment effect was markedly reduced when the ends of the bars delineated the vertical and movement of the adjustable bar delineated the oblique direction at right angles to the bars, but was negligible when the bars delineated the oblique direction and movements delineated the vertical (Experiment 3). Whereas the inner vertical ends of the bars were clearly implicated in the misalignment effect, the outside ones were not (Experiment 4).

Various issues requiring further consideration arise from these data. The first is the relationship between the apparent misalignment of oblique collinear bars (as reported here) and that of oblique collinear lines in the conventional Poggendorff figure and its variants. The misalignment effect in Figure 1B was shown earlier (Day \& Stecher, 1992) to be about the same as that in Figure 1C, and the misalignment effect in Figure $2 \mathrm{~B}$ was shown to be about the same as that in Figure $2 \mathrm{C}$ in Experiment 1 reported here. It is therefore reasonable to regard apparent misalignment between oblique collinear bars with vertical inner ends as essentially the same effect as that with similar elements abutting onto parallel vertical lines or edges. A critical feature of the stimulus figures in which the effect occurs appears to be delineation of a direction other than that of the bars themselves in the region between them. This delineation can be achieved by means of the inner edges of the ends of the bars (as in the figures described here), by extended lines or edges (as in the conventional Poggendorff figure and its variants), or by movement of one or, presumably, both elements. However, a small but statistically robust misalignment effect nevertheless occurs in the absence of any such delineation, as shown in Experiment 2. This is the second issue warranting further consideration.

From the standpoint of the perceptual-compromise explanation of apparent misalignment as set out earlier (Day \& Stecher, 1992) and summarized above in the introduction, an effect without any delineation of direction other 
than that of the bars themselves was unexpected. It is tempting to assume that the vertical is more "salient" than other directions, so that even in the absence of delineation it continues to exert an influence sufficient to bring about a slight misalignment effect. However, there is no evidence that this is so in the present context. A more likely basis for the effect without delineation is the visual anisotropy referred to as the horizontal-vertical effect ${ }^{3}$ (see Avery \& Day, 1969, 1971; Künnapas, 1955, 1957). Vertical extents for the upright observer appear slightly greater than equal horizontal extents. This effect occurs not only for two lines at right angles in the conventional HV figure viewed in the light, but also for single illuminated extents in darkness (Avery \& Day, 1969) and refers to correspondence of the extents with retinal axes rather than with the true vertical and horizontal. The apparent slightly higher elevation of the bar on the right in Figure $2 \mathrm{~A}$ compared with that in the same $45^{\circ}$ axis on the left could conceivably be due to this anisotropy; that is, the bar on the right appears to be displaced slightly upward since the vertical extent is apparently slightly greater than the horizontal component. Because this bar appears too high when truly aligned, it must be positioned lower in order to appear aligned. This could also be the basis of the slight apparent misalignment of two collinear oblique lines, the "parallel-less" Poggendorff illusion, (Day, 1973). This issue requires more detailed inquiry.

Another issue to emerge from these data is the difference between misalignment effects when the ends of the bars delineate one direction and movement of the adjustable bar delineates another. In Experiment 3, when the bar ends were vertical and movement was oblique and at right angles to the bars, the misalignment effect was about half that when both were vertical. However, when these delineations were reversed so that movement was vertical and bar ends oblique, the misalignment effect was negligible. In short, whereas vertical bar ends in conflict with oblique movement reduced the misalignment effect, oblique ends in conflict with vertical movement did not give a significant effect. This indicates that, of the two modes of delineation of direction, edges are a stronger cue than direction of movement.

A fourth issue is the bearing that the results from these experiments have on the explanation of apparent misalignment set out previously (Day, 1989; Day \& Stecher, 1992) and summarized in the introduction above. The essence of this explanation is that apparent misalignment represents a compromise in perception between alignment of elements (lines, bars, or edges) in one axis and their displacement from each other (i.e., their misalignment) in respect of another. The data from Experiment 4 are especially relevant to this explanation. It could reasonably be expected that the outer ends of the bars would serve equally with the inner ends of the $25 \times 5 \mathrm{~mm}$ bars in delineating the vertical relative to which the bars are dis- placed from each other. The finding that they do not and that, indeed, they appear to be uninvolved in the misalignment effect indicates that this explanation must be modified to take into account these findings. It is now conceivable that misalignment effects are associated only with delineation of direction by immediately adjacent, rather than more remote, edges. This is clearly an issue to be pursued.

The main outcome of these experiments is the demonstration that delineations of direction by both the inner edges of the bars and the movement of one bar during adjustments to apparent alignment interact to determine the magnitude of apparent misalignment. In the absence of any delineation by these two features, a slight effect persists, possibly due to the horizontal-vertical anisotropy of perceived extent.

\section{REFERENCES}

Avery, G. C., \& DAY, R. H. (1969). Basis of the horizontal-vertical illusion. Joumal of Experimental Psychology, 81, 376-380.

AverY, G. C. DAY, R. H. (1971). Relationship between the horizontal-vertical illusions for velocity and extent. Joumal of $E x$ perimental Psychology, 89, 22-31.

Cornsweet, T. N. (1962). The staircase method in psychophysics. American Journal of Psychology, 75, 485-491.

DAY, R. H. (1973). The oblique line illusion: The Poggendorff effect without parallels. Quarterly Joumal of Experimental Psychology, 25, 535-541.

DAY, R. H. (1989). Natural and artificial cues, perceptual compromise and the basis of veridical and illusory perception. In D. Vickers \& P. L. Smith (Eds.), Human information processing: Measures, mechanisms and models. London: Elsivier.

DAy, R. H., \& Stecher, E. J. (1992). Some variant forms of the Poggendorff illusion and their implications for an explanation. Bulletin of the Psychonomic Society, 30, 26-28.

KüNNAPAs, T. M. (1955). An analysis of the "vertical-horizontal illusion." Journal of Experimental Psychology, 49, 134-140.

KünNAPAs, T. M. (1957). The vertical-horizontal illusion and the visual field. Journal of Experimental Psychology, 53, 405-407.

Mori, T. (1981). Reduction of the Poggendorff effect by the motion of oblique lines. Perception \& Psychophysics, 29, 15-20.

Weintraub, D. J., Krantz, D. H., \& Olson, T. P. (1980). The Poggendorff illusion: Consider all the angles. Journal of Experimental Psychology: Human Perception \& Performance, 6, 718-725.

\section{NOTES}

1. The suggestion to undertake this experiment was made by Peter Wenderoth, whose contribution is gratefully acknowledged.

2. To test the possible effect of the vertical contours of the screen, a control experiment was carried out with and without the mask's defining the circular field. The figure used was the same as Figure $4 \mathrm{~A}$, and the subjects adjusted the bar on the right to apparent alignment. The mean misalignment effects were negligible (as in Experiment 4) and not different from each other. It can be concluded that screen contours did not affect apparent alignment of the bars.

3. We thank an anonymous reviewer for the suggestion that the horizontal-vertical effect might be the basis of the misalignment effect in the absence of vertical delineation.

(Manuscript received April 21, 1992;

revision accepted for publication November 16, 1992.) 